Check for updates

Cite this: RSC Adv., 2020, 10, 38831

Received 2nd October 2020 Accepted 15th October 2020

DOI: 10.1039/dOra08419k

rsc.li/rsc-advances

\section{Variation in surface properties, metabolic capping, and antibacterial activity of biosynthesized silver nanoparticles: comparison of bio-fabrication potential in phytohormone-regulated cell cultures and naturally grown plants $\uparrow$}

\begin{abstract}
Tariq Khan (D) *ab and Gul Shad Ali (iD *bc
We compared surface properties, metabolic capping and antibacterial activity of silver nanoparticles, synthesized through extracts of cell cultures of Fagonia indica and its naturally grown form. Extracts from cell cultures (produced with thidiazuron (TDZ) or melatonin (MLN)) were compared to the naturally grown whole plant extracts (WPEs) for their reducing potential, and their effects on physical and biochemical properties of the biosynthesized silver nanoparticles. UV-Vis spectroscopy revealed that the surface plasmon resonance peaked at $\lambda=415 \mathrm{~nm}$ for MLN-AgNPs, $\lambda=430 \mathrm{~nm}$ for TDZ-AgNPs and $\lambda=$ 460-465 nm for WPE-AgNPs. Transmission electron microscopy and energy dispersive X-rays of AgNPs showed that compared to WPE-AgNPs (mean diameter $=22 \mathrm{~nm}$ ), extracts from MLN- and TDZ-induced cell cultures produced particles with spherical shapes and smaller diameters (i.e. mean diameter $=15 \mathrm{~nm}$ and $19 \mathrm{~nm}$, respectively). Size distribution analysis also showed that TDZ-AgNPs were nearer to a symmetric distribution in terms of diameter (skewness $=0.80$ ) as compared to WPE-AgNPs (skewness $=0.9$ ) and MLN-AgNPs (skewness = 1.4). Furthermore, $M L N$-induced cell culture extracts produced AgNPs in higher concentration $\left(210 \mu \mathrm{g} \mathrm{mL} \mathrm{m}^{-1}\right.$ ) compared to AgNPs from TDZ-induced cell culture extracts $\left(160 \mu \mathrm{g} \mathrm{mL}^{-1}\right)$ and WPE $\left(138 \mu \mathrm{g} \mathrm{mL}^{-1}\right)$. Two-way comparisons of LC-MS/MS profiles of TDZAgNPs, MLN-AgNPs, and WPE-AgNPs revealed differences in their secondary metabolite profiles, which might account for differences in their differential response in bio-fabrication, and size distribution. Activity against different pathogenic bacterial strains, Escherichia coli, Bacillus cereus, Xanthomonas citri, Agrobacterium tumefaciens, Streptomyces griseus, and Erwinia carotovora suggested that MLN-AgNPs were more effective compared to TDZ- and WPE-AgNPs. These results indicated that phytohormones induced cell cultures can enhance the production, physical and biochemical properties of AgNPs.
\end{abstract}

\section{Introduction}

Biosynthesis of nanoparticles has been extensively explored through the use of different living systems, notably plants. Plant-based bio-reduction of metal salts is a viable, less toxic, and environmentally friendly method for the synthesis of bioactive metal nanoparticles. ${ }^{1}$ The bio-reduction potential of many different plants has been studied for the synthesis of various types of metal nanoparticles, notably silver

\footnotetext{
${ }^{a}$ Department of Biotechnology, University of Malakand, Chakdara Dir Lower, 18800, Pakistan. E-mail: tariqkhan@uom.edu.pk; Tel: +92 3339546605

${ }^{b}$ Plant Molecular and Cell Biology, Department of Plant Pathology, Mid-Florida Research and Education Center, University of Florida/Institute of Food and Agricultural Sciences, Apopka, FL, USA. E-mail: gulali@eukaryotech.com ${ }^{\circ}$ EukaryoTech LLC., Apopka, FL 32703, USA. Tel: +19706820484

$\dagger$ Electronic supplementary information (ESI) available. See DOI: $10.1039 /$ d0ra08419k
}

nanoparticles (AgNPs), which in addition to their diverse applications are considered less toxic to plants, animals, and humans. ${ }^{1,2}$ The biosynthesis of AgNPs with tissue extracts from naturally grown whole plants have also been extensively studied..$^{3-13}$ Secondary metabolites and enzymes have been linked with the biosynthesis of metallic nanoparticles. ${ }^{14,15}$ However, there are concerns regarding non-uniform biofabrication, size, and yield of AgNPs. Changes due to weather and geography and intra-planta variations in the reduction potential of plant tissues could be one of the limiting factors in providing a stable and viable source for the biosynthesis of nanoparticles. These metabolic variations could also result in inconsistent quality and quantity of nanoparticles, which in turn can lead to inconsistent bioactivities as well as a loss of market confidence. These issues require developing feasible and on-demand sources of biological extracts that can reduce silver nitrate $\left(\mathrm{AgNO}_{3}\right)$ into AgNPs. In vitro plant cultures especially cell cultures, which can be easily established and 
produced by manipulating defined plant growth regulators, might serve as an alternative. The rapid, cost-effective production of plant cell cultures through supplementation with phytohormones provides the best material for nanoparticles biosynthesis.

Using medicinal plants for establishing the cell cultures could impart medicinal potential to the biosynthesized AgNPs. Fagonia indica is a medicinal plant, which belongs to the family Zygophyllaceae. F. indica is especially known for its anticancer, ${ }^{\mathbf{1 6}}$ antidiabetic, ${ }^{17}$ anti-inflammatory, ${ }^{8}$ antimicrobial, analgesic, ${ }^{18}$ and hepato-protective ${ }^{19}$ activities. Importantly, the cell cultures of $F$. indica have shown enhanced antioxidant activities, thus providing the reducing potential for the synthesis of nanoparticles. ${ }^{20}$ Bulk quantities of $F$. indica cells can be quickly produced by supplementing the cultures with appropriate phytohormones. ${ }^{21}$ Several recent studies highlighted the role of different classes of plant secondary metabolites such as terpenoids, phenols, and alkaloids in the bio-reduction of silver salts. $^{\text {22-24 }}$ The production of these metabolites can be streamlined in cell cultures using appropriate in vitro culture conditions and media composition including variation in supplemented phytohormones. ${ }^{21}$ Alterations of growth regulators i.e. phytohormones can produce cell cultures, which can be used for producing nanoparticles with uniform size, higher yield, and desired physical and biochemical properties. There are very few reports using the manipulation of cell cultures for the biosynthesis of nanoparticles. Research on discovering the right combination of phytohormones and culture conditions will increase the use of this economical, feasible, and viable method. ${ }^{25}$ In this study, we assessed the potential of in vitro grown cell cultures of $F$. indica for the biosynthesis of AgNPs. The major objectives of our studies were (1) to test the effect of different phytohormones on the bio-fabrication potential of cell cultures of $F$. indica, (2) to compare naturally grown plant extracts (to be called whole plant extract i.e. WPE henceforth) to the cell cultures on the physical and biochemical properties of AgNPs, and (3) to compare the variation in metabolic profile of AgNPs synthesized from cell cultures and WPE.

\section{Materials and methods}

\subsection{Cell cultures and extract preparation}

The aerial parts of the $F$. indica, collected from Khyber Pakhtunkhwa Pakistan, were dried and the seeds were germinated for production of cell cultures. Stem cuttings ( $5 \mathrm{~mm}$ approx.) from a 30-days-old seed-derived plantlet were transferred to Murashige and Skoog basal medium (MS0, 1962; Phytotechnology Labs, USA) constituted as 3\% (w/v) sucrose and $0.8 \%$ (w/v) agar (Phytotechnology Labs, USA) and supplemented with $1 \mathrm{mg} \mathrm{L^{-1 }}$ of thidiazuron (TDZ). Furthermore, for analyzing the effects of melatonin (MLN) on the AgNPs synthesis potential of cell cultures of $F$. indica, MLN ( $\left.1 \mathrm{mg} \mathrm{L}^{-1}\right)$ was added to the MS media. The cultures were initiated under the following condition; $25 \pm 2{ }^{\circ} \mathrm{C}$ under $16 / 8 \mathrm{~h}$ (light/dark) photoperiod (irradiance $\approx 40 \mu \mathrm{mol} \mathrm{m} \mathrm{m}^{-2} \mathrm{~s}^{-1}$, Philips TLD 35 fluorescent lamps). The cells were sub-cultured after 3 weeks to produce the required quantity of material for AgNPs synthesis.
Aerial parts of naturally grown $F$. indica and its cell cultures, which were grown in vitro, were dried and powdered for metabolite extraction. The WPE and extract from cell cultures were prepared by boiling 5 grams of each sample in $50 \mathrm{~mL}$ of water for 10 minutes. The aqueous solutions were cooled down to room temperature and then centrifuged for 10 minutes on $6500 \mathrm{rpm}$ at room temperature. The supernatants were taken in already weighed Eppendorf tubes and subject to vacuum evaporation to determine the exact mass of the extracts. The final concentrations of extract stocks were adjusted to $50 \mathrm{mg} \mathrm{mL}$ for AgNPs synthesis. The extract stocks were stored at $4{ }^{\circ} \mathrm{C}$ until further use.

\subsection{Biosynthesis of silver nanoparticles (AgNPs)}

AgNPs were synthesized by mixing $\mathrm{AgNO}_{3}$ with WPE and extracts from TDZ- and MLN induced cell cultures, respectively as $1: 1$ at room temperature $\left(25 \pm 3{ }^{\circ} \mathrm{C}\right)$. For all the experiments, $2 \mathrm{mM} \mathrm{AgNO}_{3}$ was used with varying concentrations of the extracts as described by Ali et al. ${ }^{26}$ Suitable extract concentration for AgNPs synthesis was determined by using a two-fold dilution of crude extracts ( 50 to $0.78 \mathrm{mg} \mathrm{mL}^{-1}$ ). For time-course analysis; the extract, in optimum concentration, was mixed with $2 \mathrm{mM}$ $\mathrm{AgNO}_{3}$ in Eppendorf tubes as $1: 1$ and the reaction mixtures were kept for different periods (0 $\mathrm{min}, 5 \mathrm{~min}, 15 \mathrm{~min}, 30 \mathrm{~min}$, 1 h, 2 h, 4 h, 8 h).

\section{3. $\mathrm{Ag}^{+}$to $\mathrm{AgNP}$ conversion efficiency calculation}

To determine the yield of AgNPs in response to increasing concentration of extracts, we determined the conversion efficiency (CE) of $\mathrm{AgNO}_{3}$ to AgNPs by the extracts. Briefly, the method used was as follows. First, an empty falcon tube was weighed. Then, $20 \mathrm{~mL}$ suspensions of AgNPs were centrifuged at $14000 \times g$ for 10 minutes to pellet out AgNPs in the tube. This process was repeated three times. The pellets were then dried in a an Eppendorf extractor and the tube was weighed to determine the final weight of the AgNPs. The percent CE was calculated using the following formula:

$$
\mathrm{CE}=(\text { weight } \text { of } \mathrm{AgNPs} / \mathrm{Ags}) \times 100
$$

where, "Ags" mean input weight of $\mathrm{Ag}^{+}$ions.

\subsection{Characterization of biosynthesized AgNPs}

The formation of AgNPs was confirmed by reading the UV-Vis optical density (OD) of the samples having $\mathrm{AgNO}_{3}$ mixed with TDZ- and MLN-induced cell cultures and naturally grown $F$. indica (Synergy $^{\mathrm{TM}}$ H1 Hybrid multi-mode microplate reader BioTek()). The OD was recorded at wavelength range $(\lambda=300-$ $700 \mathrm{~nm}$ ) with readings taken every $5 \mathrm{~nm}$. Similarly, time course kinetics of AgNPs synthesis was also monitored through reading UV-Vis OD after $0 \mathrm{~min}, 5 \mathrm{~min}, 15 \mathrm{~min}, 30 \mathrm{~min}, 1 \mathrm{~h}, 2 \mathrm{~h}$, $4 \mathrm{~h}$, and $8 \mathrm{~h}$ of incubation. The ODs for these reaction mixtures were analyzed for the concentration of AgNPs to compare their yield. For further analysis, after suitable reaction time, the biosynthesized AgNPs were washed to remove residual $\mathrm{AgNO}_{3}$ through centrifugation five times at $14000 \times g$ for 10 minutes. 
The diameter, morphology and elemental analysis of the biosynthesized AgNPs were performed through a transmission electron microscope (FEI Technai F30, TEM). For TEM and energy dispersive X-ray (EDX) analyses, an aqueous suspension of AgNPs $(2 \mu \mathrm{L})$ was dropped onto 400 mesh carbon-coated copper grids and was left to dry under a lamp to form a thin layer. Afterward, the samples were subject to the TEM wherein the interaction of transmitted electrons and the AgNPs formed images that were recorded. Furthermore, EDX of the samples was also carried out to study the elemental composition of the AgNPs. The morphology analysis of AgNPs was performed through the ImageJ software. The diameter of approximately 100 particles was measured and the range of diameters of MLN-, TDZ- and WPE-AgNPs was defined. Similarly, the average diameter was also calculated from the data for comparison. Furthermore, all the data on diameter was subject to statistical analysis for size distribution pattern and finding out the polydispersity of AgNPs synthesized from different sources.

\subsection{Metabolite profiling of AgNPs using LC-MS/MS}

For LC-MS/MS analysis, AgNPs were synthesized using WPE of F. indica (termed WPE-AgNPs), TDZ-grown cell cultures (TDZAgNPs), MLN-grown cell cultures (MLN-AgNPs) as mentioned above. The three types of AgNPs and the extracts alone (labeled WPE, TDZ, and MLN) were centrifuged at $14000 \times g$ for 5 minutes. The supernatants were discarded and the AgNPs pellets and, extract-only tubes, were washed with distilled water followed by centrifugation at $14000 \times g$ for 5 minutes. This procedure was repeated five times. The pelleted AgNPs and the control (extract-only tubes) were resuspended in $250 \mu \mathrm{L} 100 \%$ methanol through ultrasonication for 30 seconds. The samples were centrifuged again at $10000 \times g$ for $10 \mathrm{~min}$ and the methanol supernatants were transferred to sterilized empty Eppendorf tubes. These samples were analyzed using LC-MS/ MS according to the detailed protocol (ESI).

\subsection{Anti-bacterial microdilution assays of biosynthesized AgNPs}

The bactericidal potential of biosynthesized AgNPs was assessed against two human pathogens Escherichia coli and Bacillus cereus, and four plant pathogens Xanthomonas citri, Agrobacterium tumefaciens, Streptomyces griseus, and Erwinia carotovora. The antibacterial activity was determined through the broth microdilution assay defined by Wiegand et al., 2008. ${ }^{27}$ Briefly, two-fold dilutions (250 to $0.24 \mu \mathrm{g} \mathrm{mL} \mathrm{m}^{-1}$ ) of prewashed AgNPs were prepared in a microtiter plate (CytoOne, CAT \#CC7672-7596). The bacterial strains were grown overnight at $28{ }^{\circ} \mathrm{C}\left(=37^{\circ} \mathrm{C}\right.$ for $E$. coli $)$ on solidified broth to obtain single colonies. Uniform colonies were suspended in liquid broth and their $\mathrm{OD}_{625} \mathrm{~nm}$ was adjusted between 0.08 to 0.13 . The bacterial suspensions were then diluted to 1 : 100 to obtain $1 \times 10^{8} \mathrm{CFU}$ per $\mathrm{mL}$ as a final bacterial suspension. Each well of the microtiter plate was inoculated with $50 \mu \mathrm{L}$ of the bacterial suspension. The reaction well without AgNPs and containing only LB and the bacterial suspension was used as growth control (positive control). Similarly, reaction well containing only LB media was used as sterility control (negative control). The plates were incubated stationary at $28{ }^{\circ} \mathrm{C}\left(=37{ }^{\circ} \mathrm{C}\right.$ for $E$. coli $)$ for 24 hours, maintaining complete humidity to avoid evaporation of the reaction mixtures. Activity was assessed by measuring the absorbance of the suspension spectrophotometrically $\left(\mathrm{OD}_{625}\right.$ $\mathrm{nm}$ ). Minimum inhibitory concentration (MIC) was defined as the least concentration of AgNPs that completely inhibited bacterial growth.

\subsection{Mechanism of bacterial inhibition by the biosynthesized AgNPs}

The oxidant-sensitive probe $2^{\prime}, 7^{\prime}$-dichlorodihydrofluorescein diacetate $\left(\mathrm{H}_{2}\right.$ DCFDA) was used to determine the intracellular levels of reactive oxygen species (ROS) in cells treated with different concentrations of AgNPs $\left(0.5-250 \mu \mathrm{g} \mathrm{mL}^{-1}\right)$. A slightly modified protocol of Pérez et al. (2007) was followed for ROS quantification. $^{28}$ Pérez et al., (2007) used a pre-coated LB medium with tellurite while our protocol involved the addition of AgNPs once the bacterial were treated with $\mathrm{H}_{2}$ DCFDA properly and then AgNPs were applied. Briefly, the modified protocol is given here. Bacterial cells were grown in LB medium until $\mathrm{OD}_{600}$ reached 0.5 . The bacterial cells were pelleted through centrifugation $(500 \times g)$ and washed with $10 \mathrm{mM}$ potassium phosphate buffer ( $\mathrm{pH}$ 7.0). The pellet was re-suspended in the same buffer by gentle vortexing. Then, $10 \mathrm{mM} \mathrm{H}_{2}$ DCFDA at a ratio of $1: 2000$ was added, and the mixture was incubated at $37{ }^{\circ} \mathrm{C}$ for 30 minutes at $50 \mathrm{rpm}$. Bacterial cells were washed two times with the same buffer to remove extracellular $\mathrm{H}_{2}$ DCFDA. The cleaned cells were suspended in the same buffer and treated with a series of AgNPs concentrations (0, 1.95, 3.9, 7.81, $15.62,31.25,62.5,125,250$, and $500 \mu \mathrm{g} \mathrm{mL}^{-1}$ ). The fluorescence intensity of DCF was recorded after $30 \mathrm{~min}$ using the BioTek multimode microplate reader at excitation/emission $\lambda=488$ / $535 \mathrm{~nm}$.

\subsection{Statistical analysis}

All the experiments were run in triplicate. Regression analysis was performed for the TEM results. Similarly, chi-square analysis was performed to assess the goodness of fit of the values. Furthermore, curve fitting was performed for analysis of asymmetry in the size distribution of nanoparticles.

\section{Results and discussion}

3.1. Comparative analysis of bio-fabrication potential of thidiazuron and melatonin induced cell cultures and naturally grown $\boldsymbol{F}$. indica

Higher absorbance in the wavelength range between $\lambda=400 \mathrm{~nm}$ and $\lambda=500 \mathrm{~nm}$ is a characteristic property of AgNPs. We compared AgNPs synthesis using WPE and cells grown in the presence of TDZ, and MLN, using UV-Vis spectroscopy. Sharper and higher peaks in the $\lambda=400-500 \mathrm{~nm}$ range were observed for AgNPs synthesized with phytohormones induced cell culture extracts compared to WPE (Fig. 1). This can also be correlated with the relative uniformity of MLN- and TDZ-AgNPs compared to WPE-AgNPs. As can be seen in Fig. 5, despite the generally 


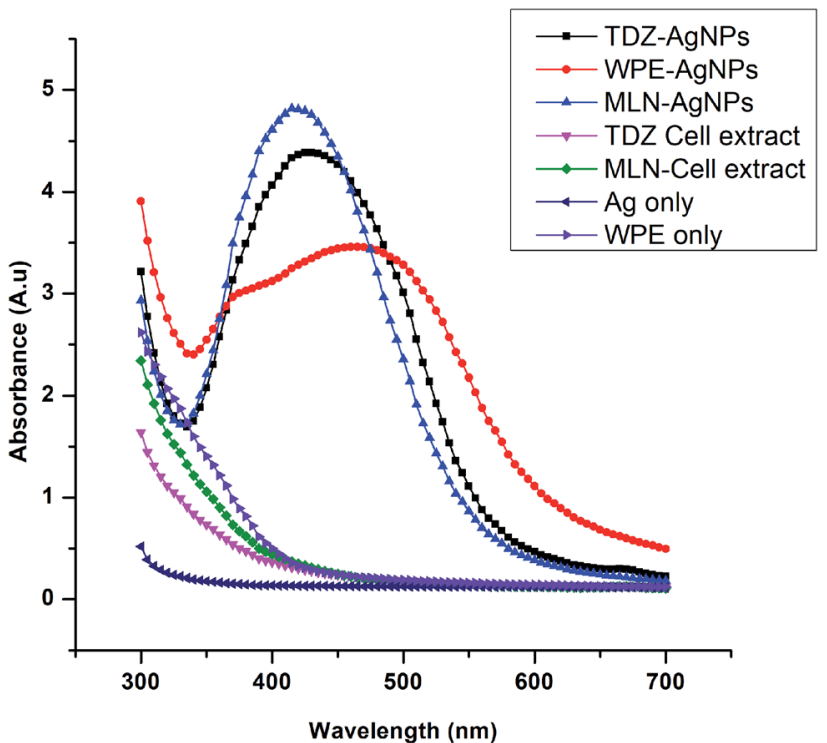

Fig. 1 Comparative surface plasmon resonance peaks of reaction mixtures for AgNPs synthesis in WPE, and extracts from TDZ-induced cell cultures, and MLN-induced cells cultures. Controls; cell extracts only, Ag, and WPE only.

higher degree of variation in sizes and shapes, the MLN-AgNPs (Fig. 5a) and TDZ-AgNPs (Fig. 5b) are more uniformly sized and shaped compared to WPE-AgNPs (Fig. 5c). This suggests an enhanced synthesis of uniform size AgNPs through cell culture extracts. ${ }^{29}$ Information about physical state, including diameter and morphology, can be obtained easily by analyzing the spectral properties of AgNPs due to their unique optical properties. ${ }^{30}$ UV-Vis spectroscopy revealed that for reactions with cell extracts, the UV-Vis absorption peaks were in the range of $\lambda=$ 410-435 nm for TDZ-AgNPs and MLN-AgNPs. Whereas in the case of WPE-AgNPs, the UV-Vis absorption peaks were in the range of $\lambda=455-475 \mathrm{~nm}$. The UV-Vis absorption peaked at $\lambda=$ $415 \mathrm{~nm}$ for MLN-AgNPs, $\lambda=430 \mathrm{~nm}$ for TDZ-AgNPs and $\lambda=$ 460-465 nm for WPE-AgNPs. Further, it was observed that UVVis absorption peaks of WPE-AgNPs lied towards longer wavelength range and were broader compared to UV-Vis absorption peaks of TDZ-AgNPs and MLN-AgNPs, which were narrow (Fig. 1). The UV-Vis absorption peak shifted towards shorter wavelengths and become narrower in MLN-AgNPs compared to TDZ-AgNPs, indicating the formation of smaller diameter AgNPs. ${ }^{31}$ The reason for this is stated to be the observation that the spectral response of AgNPs is a function of their diameter. As the diameter of AgNPs increases, the UV-Vis absorption peaks shifts towards longer wavelengths and broadens. ${ }^{30}$ The wide and low-intensity bands of the WPE-based reaction mixture suggest the presence of polydisperse AgNPs with a wider size range. ${ }^{32}$ Polydispersity is suggested to be due to the presence of a variety of secondary metabolites. ${ }^{26}$ Due to the complexity of cell types and metabolic activity of naturally grown whole plants, the diversity of secondary metabolites in WPE is likely to be higher than in cell cultures, which consist of uniform cell types, culture conditions, and metabolic activities.
The rapid reduction of silver nitrate and formation of AgNPs in cell culture extracts can be linked with the abundance of important secondary metabolites in cell cultures of $F$. indica triggered by the application of the phytohormones, i.e. TDZ and MLN. $^{21}$

Bio-fabrication of AgNPs using extracts from cell cultures grown in vitro has been less explored. Only a few studies are available which suggests that AgNPs synthesized using callus extract possess greater stability. ${ }^{33}$ The greater stability can be attributed to metabolically active, rapidly dividing plant cells which usually produce higher amounts and uniform classes of secondary metabolites. ${ }^{34}$ Some of these metabolites include polyphenolic compounds with higher reducing potential. Synthesis of nanoparticles through phenolic compounds such as flavonoids likely occurs via their tautomeric transformations from the enol- to the keto-form, which release a reactive hydrogen atom that reduces metal ions to form nanoparticles. ${ }^{35}$

\subsection{Dose-response in TDZ-, MLN- and WPE-AgNPs synthesis}

We observed a positive correlation between AgNPs synthesis and the concentration of cell culture extracts and WPE. The UVVis analysis of AgNPs synthesized through WPE and extracts of cell culture showed that the yield of AgNPs increased with increasing extract concentration up to $25 \mathrm{mg} \mathrm{mL}^{-1}$ (Fig. 2). The highest average yields of AgNPs at extract concentration of $25 \mathrm{mg} \mathrm{mL}{ }^{-1}$ were recorded in reaction mixtures yielding MLNAgNPs $\left(173.09 \mu \mathrm{g} \mathrm{mL}{ }^{-1}\right)$ compared to TDZ-AgNPs $(148.5 \mu \mathrm{g}$ $\left.\mathrm{mL}^{-1}\right)$ and WPE-AgNPs $\left(127.02 \mu \mathrm{g} \mathrm{mL}^{-1}\right)$ after 4 hours of reaction initiation. Regression analysis of data obtained from cell cultures and WPE mediated biosynthesis showed that one curve does not fit all the data sets. Therefore, different curves were defined for each data set as the results of TDZ-, MLN- and WPEAgNPs were significantly different ( $p$-value $<0.0001)$. The concentration of reducing metabolites in the extracts has been demonstrated as an important contributing factor to

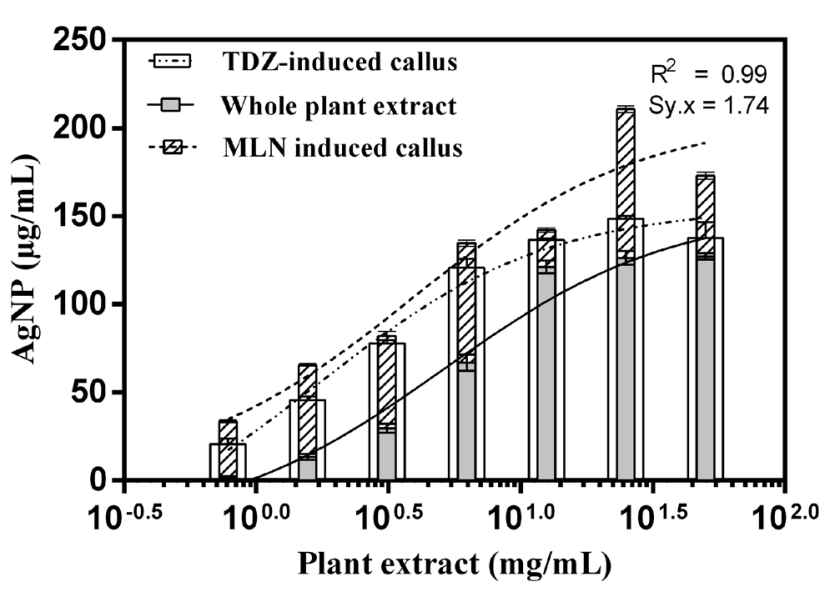

Fig. 2 Dose-response recorded in terms of AgNPs yield when supplemented with different concentrations of WPE and extracts from TDZ-induced cell cultures, and MLN-induced cell cultures at a constant concentration of $\mathrm{AgNO}_{3}(2 \mathrm{mM})$. 
determining the size and distribution of silver nanoparticles. ${ }^{36}$ At lower or higher concentration of the extract, the silver ions released from $\mathrm{AgNO}_{3}$ through reduction, result in the formation of grayish color and agglomerated AgNPs. Moderate concentrations of plant extract result in the synthesis of uniform colloidal AgNPs. Increasing the concentration of extracts results in high localized amounts of reducing agents, which leads to the formation of larger clusters or macromolecules and thus the agglomeration of AgNPs. ${ }^{26}$

\subsection{Reaction kinetics of AgNPs biosynthesis}

To determine the effects of reaction time on the saturation limits of AgNPs synthesis, time-course analysis ranging from 0 to $8 \mathrm{~h}$ were conducted. These analyses showed that with both cell culture extracts and WPE, AgNPs synthesis begins rapidly in the presence of daylight. In terms of AgNPs yield, all the reaction mixtures extracts showed a typical chemical kinetic reaction. For reactions containing extracts from MLN-induced cell cultures, AgNP synthesis continued to increase even after $8 \mathrm{~h}$ of reaction initiation while the reaction mixture for the synthesis of TDZ- and WPE-AgNPs reached saturation after $4 \mathrm{~h}$ of reaction initiation, and the concentration curves were either plateaued or declines (Fig. 3). After $8 \mathrm{~h}$ of reaction initiation, the concentration of MLN-AgNPs was the highest $\left(210.7 \mu \mathrm{g} \mathrm{mL}{ }^{-1}\right)$, followed by TDZ-AgNPs $\left(160 \mu \mathrm{g} \mathrm{mL}^{-1}\right)$ compared to WPE-AgNPs (138 $\mu \mathrm{g} \mathrm{mL} \mathrm{mL}^{-1}$ ) (Fig. 3). The role of MLN in enhancing bioreduction potential is likely associated with the production of polyphenolic compounds and alkaloids. Sheshadri et al. related the enhanced bio-reduction potential and the production of unique flavonoids in leaves of Catharanthus roseus treated with MLN. ${ }^{37}$ The role of both exogenous and endogenous MLN has been studied extensively in plants. MLN ( $N$-acetyl-5methoxytryptamine) is a particularly important hormone found both in mammals and plants. Phytomelatonin, i.e. the

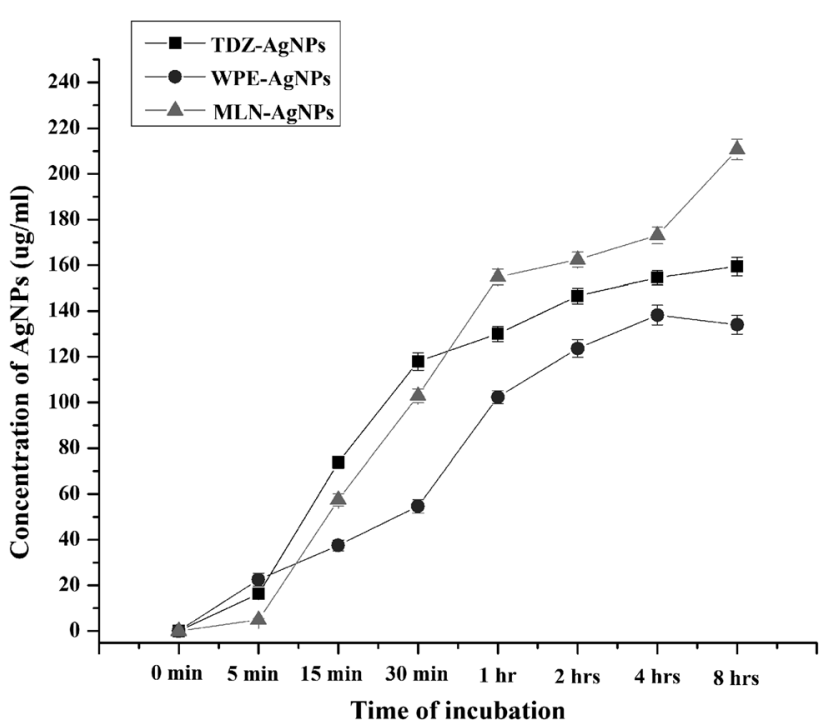

Fig. 3 Time course analysis of yield of AgNPs biosynthesized through extracts of TDZ-induced cell cultures, MLN-induced cell cultures, and whole plant of $F$. indica. endogenously produced MLN affects the plant's physiology in a range of ways including improvements in photosynthesis efficiency, metabolisms of carbohydrates, lipids, nitrogen compounds, interventions in the regulation of all plant hormones, involvement secondary metabolism, and triggering defense responses. Arnao et al., (2020) have reviewed extensively the importance of phytomelatonin. ${ }^{38}$ To this end, the application of exogenous MLN could potentially trigger these responses. Exogenous MLN upregulates the antioxidant system of plants to protect them against reactive oxygen species. In this process, MLN triggers unique antioxidant secondary metabolites in plants, ${ }^{39} \mathrm{~A}$ very important response that could be triggered by exogenous MLN is the secondary metabolic responses and changes in cell metabolism to produce secondary metabolites and biomolecules of particular importance for bioreduction of $\mathrm{Ag}^{+}$to form AgNPs. This has already been demonstrated by Sheshadri et al., 2015. ${ }^{37}$ The article reported that exogenous application of MLN to plant leaves could trigger important biomolecules in those leaves that could in turn enhance their bio-reducing potential. ${ }^{37}$ In our study this could be confirmed by the unique features in the secondary metabolite profile of AgNPs biosynthesized via extracts of MLN-induced cell cultures to plants (Table S1†).

Furthermore, visual differences also revealed aggregation in WPE-AgNPs while MLN-AgNPs and TDZ-AgNPs remained in the colloidal state. Images were taken for the three reactions after 48 hours to assess the stability of AgNPs. Visual assessment of the reactions showed that AgNPs synthesized with extracts from MLN-induced cell cultures were darker and remained in a colloidal state after 48 hours of reaction initiation. The reaction mixture containing TDZ-AgNPs was also uniformly distributed with a light brown color indicating a lesser abundance of AgNPs compared to MLN-AgNPs (Fig. 4). On the other hand, agglomeration and sedimentation occurred in the WPEbased reaction, this shows the higher size and non-stable nature of WPE-AgNPs. Staying in the colloidal state is a desirable property of nanoparticles. ${ }^{22}$ The reason for these differences might be the presence of diverse classes of secondary

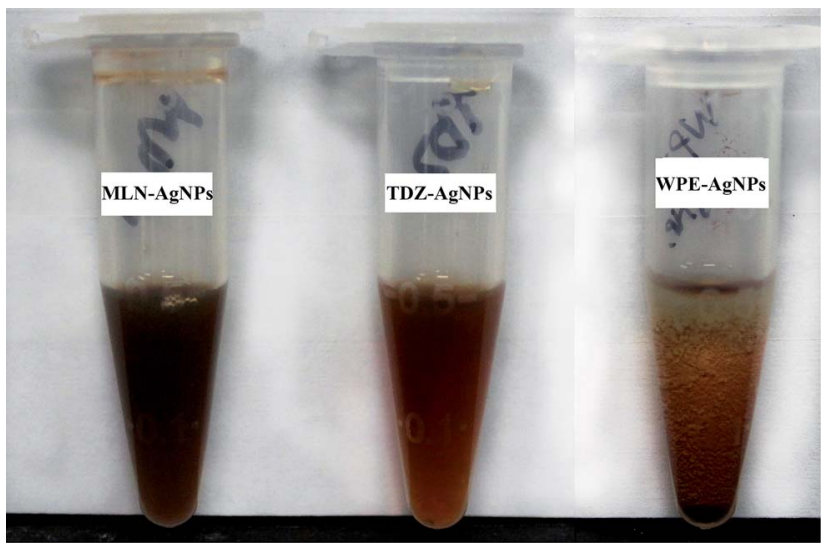

Fig. 4 Comparison of the reaction mixture of AgNPs synthesized through extracts of MLN induced cell cultures (left), TDZ-induced cell culture (center), and whole plant (right) after 48 hours of incubation. 


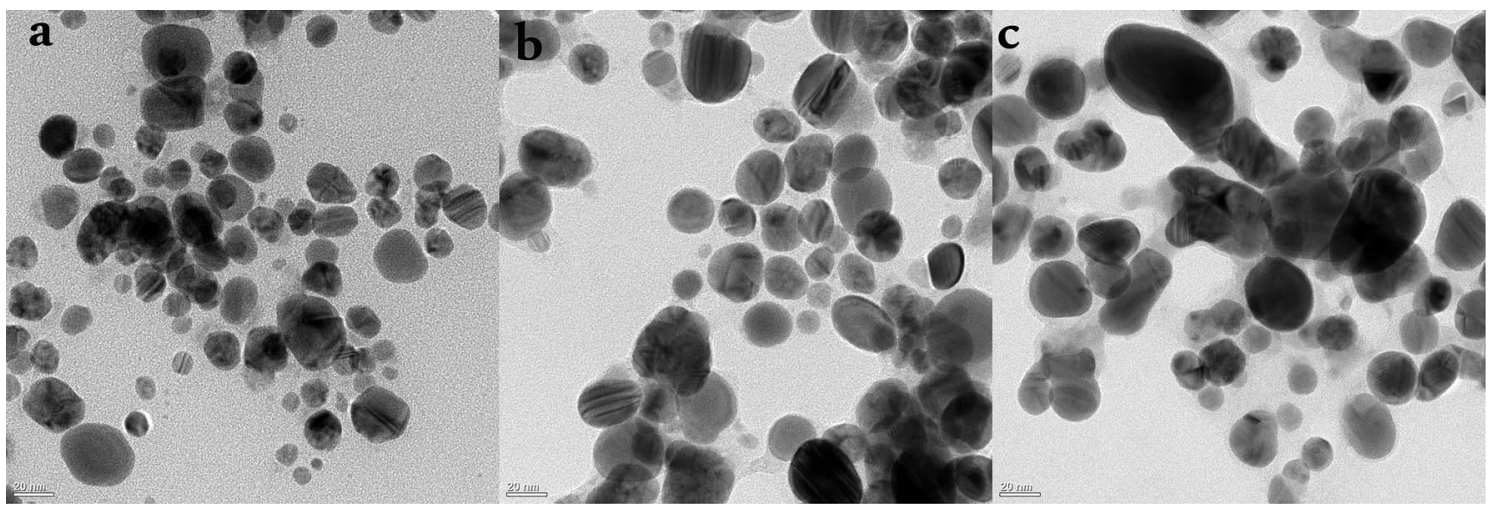

Fig. 5 Transmission electron microscopy (TEM) based images of AgNPs synthesized via extracts from (a) MLN-supplemented cell cultures (b) TDZ-supplemented cell cultures and (c) naturally grown F. indica.

metabolites in the whole plant versus uniform classes of metabolites in cell culture extracts that are involved in AgNPs synthesis.

\subsection{Transmission electron microscopy and EDX analyses of AgNPs}

Morphology analysis of various TEM images was performed through ImageJ software. ${ }^{40}$ The size distribution of AgNPs shows an interesting pattern suggesting poly-dispersal of the nanoparticles. TEM and EDX analyses were performed for assessing the impact of extract type i.e. WPE, TDZ-induced cell culture extract, and MLN-induced cell culture extract on the morphology and size distribution of AgNPs. The diameters of MLN-AgNPs, TDZ-AgNPs, and WPE-AgNPs, ranged in size from 2-36 $\mathrm{nm}$ (average $15 \mathrm{~nm}$ ), 8-52 $\mathrm{nm}$ (average $19 \mathrm{~nm}$ ), and 4$57 \mathrm{~nm}$ (average $22 \mathrm{~nm}$ ), respectively (Fig. $5 \mathrm{a}, \mathrm{b}$ and c). Analysis of the high-resolution transmission electron microscopy (TEM) images showed specific lattice fringes of Ag. Furthermore, EDX analysis for multiple AgNPs samples showed that the highest of atomic peaks were that of Ag (Fig. S1†). A closer look at Fig. S1† reveals that the highest peaks are those revealed by MLN-AgNPs (Fig. S1b $\uparrow$ ), where Ag peaked at above 3000 counts followed by
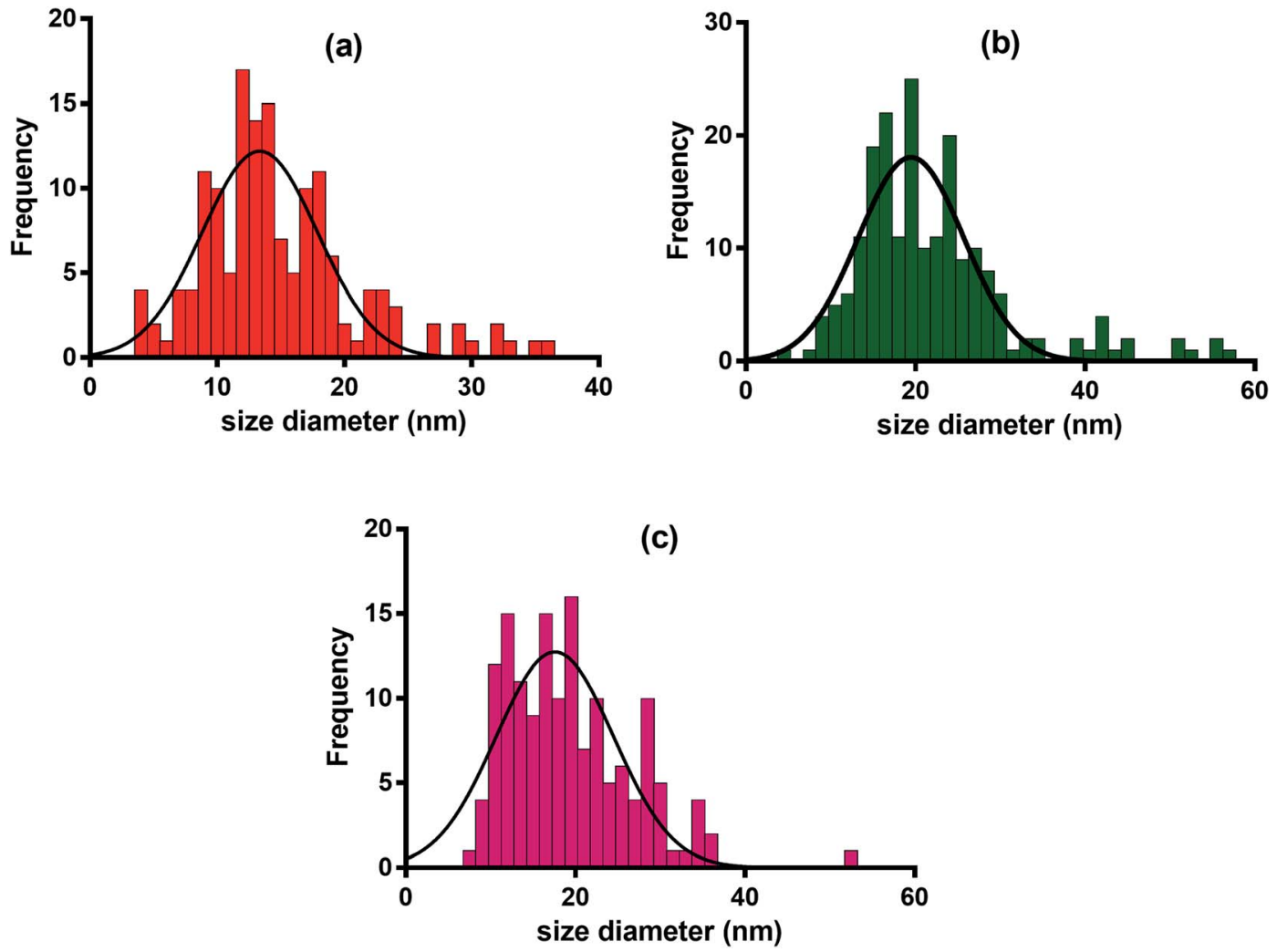

Fig. 6 Size distribution of AgNPs synthesized through aqueous extracts of (a) TDZ-induced cell cultures (b) MLN-induced cell cultures and (c) naturally grown $F$. indica. 
Ag peaks in TDZ-AgNPs which went above 1000 count (Fig. S1a $\dagger$ ) compared to Ag peaks in WPE-AgNPs which reached up to 1000 count (Fig. S1c $\dagger$ ). This further verifies more efficient biofabrication of AgNPs in MLN- and TDZ- based cell culture extracts. In addition to $\mathrm{Ag}$, other prominent peaks belonged to $\mathrm{Cu}$ and $\mathrm{Si}$, which are commonly found in nanoparticles synthesized with tissue extracts from plants. ${ }^{26}$ Peaks corresponding to $\mathrm{C}$ are routinely observed in samples deposited on C-coated copper grids.

Image J based analysis of the AgNPs revealed that there was a broad distribution of the nanoparticles. A comparison of AgNPs synthesized with cell culture extracts shows that the TDZAgNPs were uniformly distributed compared to WPE-AgNPs, which were bigger and had varying sizes and shapes. The polydispersity of WPE-AgNPs can be attributed to the diverse availability of different classes of secondary metabolites with variable reduction properties, which may affect AgNPs nucleation and growth. ${ }^{26}$ Statistical analysis of the size distribution pattern of different AgNPs showed that TDZ-AgNPs have the least skewness (0.80) and is nearer to symmetric distribution compared to WPE-AgNPs (skewness $=0.9$ ) and MLN-AgNPs (skewness $=1.4$ ) (Fig. 6). Skewness represents asymmetry in size distribution. The fact the WPE-AgNPs are more skewed could be because naturally grown plants tend to synthesize a diverse array of secondary metabolites and contain many different biomolecules with differential reduction potential to synthesize AgNPs of varying sizes. One important characteristic of the plant's secondary metabolism is diverse chemical structures. ${ }^{41}$ Plants produce an array of chemical structures that could contribute to the bio-fabrication of AgNPs. Naturally grown plants are exposed to a plethora of stressors and triggers. This calls for the need for the production of these compounds which could ultimately be the reason for the production of widely distributed AgNPs sizes. This can also be correlated with TEM images of WPE-AgNPs in Fig. 5a. In our results, TDZ-AgNPs seems to be more symmetrically distributed compared to WPEAgNPs and MLN-AgNPs (Fig. 6). The fact that MLN-AgNPs are also skewed and there are nanoparticles in range of up to $52 \mathrm{~nm}$, can be related with the triggering of the many unique compounds by MLN (Table S1 $\dagger$ ). The reason for such results even though MLN-AgNPs were produced from extracts of cell cultures grown in vitro could be inferred from the fact that MLN plays a diverse function in plants. MLN affects plants in many ways as mentioned above and defined in many studies. ${ }^{42}$ Exogenous MLN could lead to the production of a variety of secondary metabolic responses, and metabolism of other biomolecules such as photosynthetic pigments, proteins, lipids, etc. which in turn could play their role in the synthesis and biofabrication of AgNPs thus producing a diversity of AgNPs (Fig. 5a and 6b).

\subsection{LC-MS/MS profile of capping agents on AgNPs}

To determine what kind of compounds might contribute to the capping and stability of AgNPs, we conducted LC-MS/MS analyses of extracts obtained from WPE- and TDZ- and MLN-AgNPs. These analyses revealed (a total of 80 ) diverse compounds attached to AgNPs. Comparative analysis of all the samples revealed that among these compounds, there were 02 compounds detected only in the extract from TDZ-AgNPs, 30 compounds detected only in the extract from MLN-AgNPs, and only 01 unique compounds in the extract from WPE-AgNPs. Similarly, there were 14 compounds detected both in extracts from TDZ- and MLN-AgNPs but not in the extract from WPEAgNPs. While there were 23 compounds detected only in extracts from TDZ-AgNPs and WPE-AgNPs. There were 08 compounds detected to be associated only with MLN- and WPEAgNPs but not with TDZ-AgNPs. Finally, there were only 02 compounds common in all the samples (Fig. 7). Overall, out of the total of 80 compounds, there were 46 unique compounds associated with AgNPs synthesized using cell cultures extracts that were not detected in WPE-AgNPs (Table S1†). The higher number of such unique compounds is indicative of the unique metabolite of cell culture extracts, which can ultimately be related to the efficient and high stability of AgNPs synthesized via extracts from in vitro cell cultures compared to WPE-AgNPs.

Cell cultures produce sufficient amounts of secondary metabolites as the cells grow under different plant growth regulators. These results suggest that unique compounds were produced in in vitro grown cell cultures of $F$. indica were not present in extracts from naturally gown $F$. indica. While the cells can produce a diverse array of compounds, the controlled in vitro conditions and the application of a single type of phytohormone makes it possible for the cells to produce compounds which may have been responsible for stable AgNPs production. ${ }^{43}$

According to results from METLIN and cross-checking with the NCBI PubChem, it was observed that the compounds involved in the bio-fabrication of AgNPs belonged to diverse classes including amines, amino acids, polypeptides, fatty

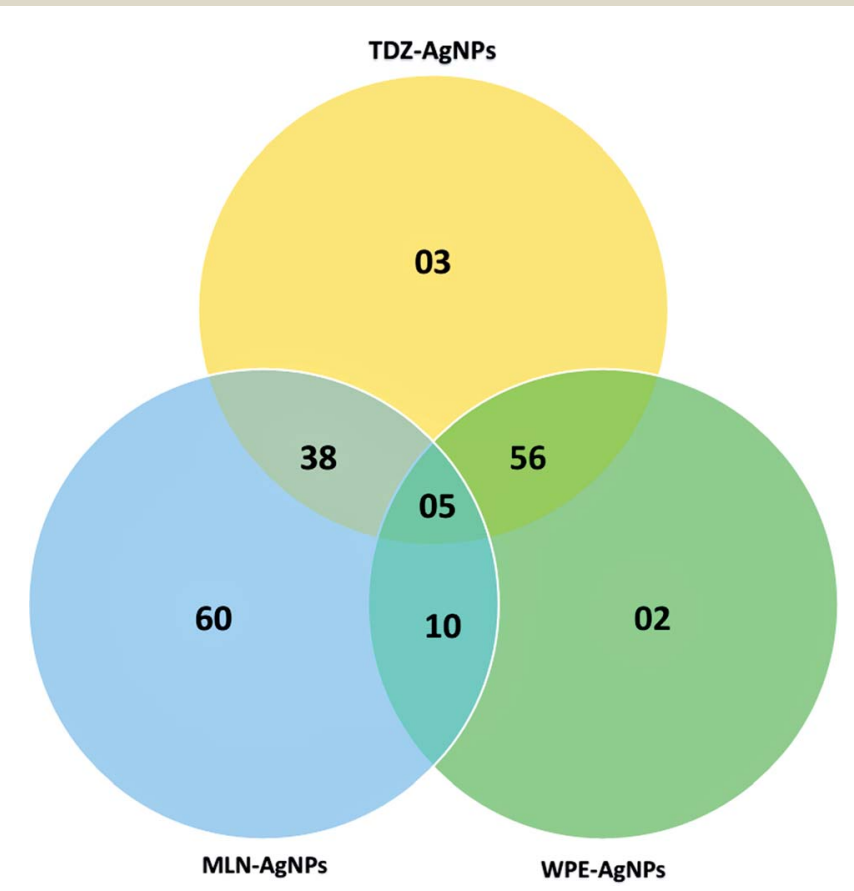

Fig. 7 Venn diagram for the comparative analysis of compounds detected through LC-MS/MS. 
acids, alkaloids, terpenes, and polyphenols. As indicated in Table S1, $\uparrow$ the predominant compounds involved in biofabrication were amine-based compounds followed by fatty acids, alkaloids, and peptides, respectively. $F$. indica is naturally rich in these metabolites especially fatty acids, aromatic amine groups, and alkaloids. ${ }^{44}$ Secondary metabolites have been shown to affect the bio-reduction of silver salts to form AgNPs. For instance, Marslin et al. stated that flavonoids are the major contributors to the green synthesis of nanoparticles. ${ }^{45}$ Flavonoids like hesperidin, naringin, diosmin have been shown to affect optical, morphological, and biochemical properties of AgNPs. ${ }^{46}$ This demonstrates that the different responses in terms of the physical and biochemical properties of AgNPs synthesized from the three extract sources can be attributed to their differential metabolite profile (Table S1†).

\subsection{Comparison of the antibacterial potential of the AgNPs}

The AgNPs synthesized with $F$. indica cell cultures and whole plant extracts were effective against different pathogenic bacterial strains, Escherichia coli, ${ }^{47}$ Bacillus cereus, ${ }^{48}$ Xanthomonas citri, ${ }^{49}$ Agrobacterium tumefaciens, ${ }^{50}$ Streptomyces griseus, ${ }^{51}$ and Erwinia carotovora. ${ }^{52} \mathrm{X}$. citri causes citrus canker, ${ }^{49} A$. tumefaciens causes crown gall disease, ${ }^{50} \mathrm{~S}$. griseus is the cause of scabies in important crop plants like potato, ${ }^{51}$ and E. caratovora is the cause of soft rot in many agriculturally and industrially important plants ${ }^{53}$ AgNPs synthesized through callus and whole plant extract showed lower MIC values against the abovementioned bacteria (Table 1). AgNPs synthesized through MLN callus extract were found more potent compared to AgNPs synthesized with extracts from callus grown with TDZ or whole plants. The differential response in terms of absorption of light at $\mathrm{OD}_{625} \mathrm{~nm}$ is shown in Fig. S2. $\dagger$ The MIC values for MLNAgNPs were approximately $1.95 \mu \mathrm{g} \mathrm{mL}{ }^{-1}$ against A. tumefaciens and E. caratovora, $3.90 \mu \mathrm{g} \mathrm{mL}^{-1}$ against $E$. coli and $S$. griseus, and $7.81 \mu \mathrm{g} \mathrm{mL} \mathrm{m}^{-1}$ against $B$. cereus and $X$. citri. Detailed data on absorption for MLN-, TDZ- and WPE-AgNPs can be found in ESI (Table S2 $\dagger$ ). Several studies have reported that biosynthesized AgNPs display higher potency and efficacy than those synthesized using chemical and physical methods. ${ }^{37}$ Our findings are also consistent with previous studies, which have demonstrated stronger antibacterial activity of AgNPs synthesized with callus extracts. ${ }^{54,55}$ For example, Xia et al. showed the potential of AgNPs synthesized with an extract from Taxus yunnanensis against Gram-negative bacteria including E. coli. ${ }^{55}$

\subsection{Demonstration of the mechanism of action of bacterial inhibition by AgNPs}

Living organisms usually produce ROS when subjected to antimicrobial compounds. For example, intracellular superoxide radicals are formed in bacteria when treated with zinc oxide nanoparticles. ${ }^{56}$ In our analyses, we also observed that MLN-AgNPs induced ROS in bacterial cells. The highest amount of ROS was induced in $S$. aureus followed by $X$. citri and $E$. caratova. Furthermore, MLN-AgNPs concentrations in the range of 1.9-31.5 $\mu \mathrm{g} \mathrm{mL} \mathrm{mL}^{-1}$ produced the highest amount of ROS; concentrations higher or lower than this range lead to lesser induction of ROS.

As expected, AgNPs provision increased ROS (Fig. 8). The major factor for the inhibition of bacteria is ROS, which is produced when AgNPs interacts with the bacterial suspension. As the AgNPs concentration increases, there is an increase in the fluorescence intensity of the bacterial cultures. The response varies for the different bacterial strains. However, it is seen that for each bacterial strain, there is a decrease in fluorescence intensity after the AgNPs exceeds specific concentrations (Fig. 8). For instance, the highest fluorescence was recorded from $S$. aureus cultures when provided with $15.6 \mu \mathrm{g}$ $\mathrm{mL}^{-1}$ AgNPs. Thus, it can be concluded that the AgNPs produced antibacterial activity through increased ROS

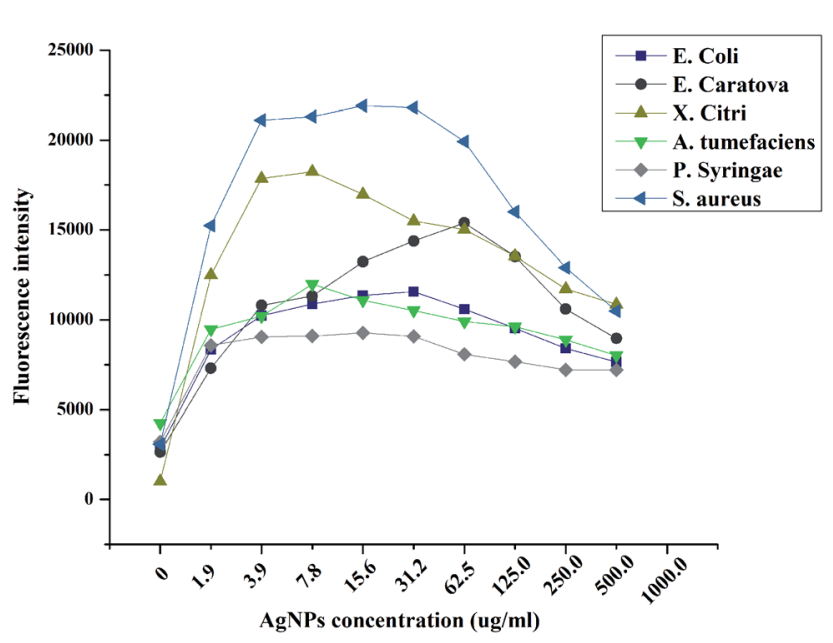

Fig. 8 Reactive oxygen quantification in bacterial cultures grown in the presence of different concentrations of AgNPs synthesized in MLN-based in vitro callus cultures.

Table 1 Minimum inhibitory concentrations of AgNPs synthesized through different types of extracts from cell cultures and naturally grown plant extract of $F$. indica

\begin{tabular}{|c|c|c|c|c|c|c|}
\hline \multirow{2}{*}{$\begin{array}{l}\text { AgNPs type } \\
\text { (based } \\
\text { on extract) }\end{array}$} & \multicolumn{6}{|c|}{ Minimum inhibitory concentration $\left(\mu \mathrm{g} \mathrm{mL}^{-1}\right)$} \\
\hline & $\begin{array}{l}\text { Escherichia } \\
\text { coli }\end{array}$ & $\begin{array}{l}\text { Bacillus } \\
\text { cereus }\end{array}$ & $\begin{array}{l}\text { Xanthomonas } \\
\text { citri }\end{array}$ & $\begin{array}{l}\text { Agrobacterium } \\
\text { tumefaciens }\end{array}$ & $\begin{array}{l}\text { Streptomyces } \\
\text { griseus }\end{array}$ & $\begin{array}{l}\text { Erwinia } \\
\text { carotovora }\end{array}$ \\
\hline MLN-AgNPs & $\leq 3.90$ & $\leq 7.81$ & $\leq 7.81$ & $\leq 1.95$ & $\leq 3.90$ & $\leq 1.95$ \\
\hline TDZ-AgNPs & $\leq 7.81$ & $\leq 31.25$ & $\leq 31.25$ & $\leq 3.90$ & $\leq 7.81$ & $\leq 3.90$ \\
\hline WPE-AgNPs & $\leq 7.81$ & $\leq 15.62$ & $\leq 31.25$ & $\leq 3.90$ & $\leq 7.81$ & $\leq 3.90$ \\
\hline
\end{tabular}


generation, which is responsible for cell membrane and DNA damage in bacteria. ${ }^{28}$

\section{Conclusion}

AgNPs synthesized through bio-reduction by extracts of phytohormone-induced cell cultures grown in vitro tends to be efficient and stable compared to the AgNPs synthesized through extracts of naturally grown $F$. indica. AgNPs synthesized through the extract of MLN-based cell cultures showed enhanced spectral properties and physical features than AgNPs synthesized with TDZ-based cell culture extract and WPE. Phytohormones trigger unique secondary metabolites in cell culture which prove effective in bio-fabrication and imparting stability to the biosynthesized AgNPs. Only a small fraction of compounds was common among all AgNPs suggesting that they perform an essential function in the synthesis or capping of AgNPs. Research is needed to explore the effects of many different phytohormones and other types of in vitro cultures on AgNPs synthesis. Similarly, other types of metal nanoparticles should also be explored through such studies to assess the best synthesis routes for potent and stable nanoparticles in an economical way.

\section{Conflicts of interest}

There are no conflicts to declare.

\section{Acknowledgements}

We are thankful to the Mid-Florida Research and Education Centre, University of Florida for providing the resources and hosting the project. We are also thankful to the Department of Chemistry, University of Florida, USA for facilitation in LC-MS/ MS analysis and to the University of Central Florida for conducting the TEM and EDX analysis. We are thankful to the Higher Education Commission, Pakistan for financial support to conduct this study. This research was supported by the International Research Support initiative program of the Higher Education Commission, Pakistan (IRSIP 31 BMS 40).

\section{References}

1 Z.-u.-R. Mashwani, T. Khan, M. A. Khan and A. Nadhman, Appl. Microbiol. Biotechnol., 2015, 99, 9923-9934.

2 A. K. Mittal, Y. Chisti and U. C. Banerjee, Biotechnol. Adv., 2013, 31, 346-356.

3 A. Miri, M. Sarani, M. Rezazade Bazaz and M. Darroudi, Spectrochim. Acta, Part A, 2015, 15, 287.

4 P. M. Mishra, S. K. Sahoo, G. K. Naik and N. Parida, Mater. Lett., 2015, 566-571.

5 B. Mohapatra, S. Kuriakose and S. Mohapatra, J. Alloys Compd., 2015, 637, 119-126.

6 M. Nasrollahzadeh, S. M. Sajadi, F. Babaei and M. Mahamd, J. Colloid Interface Sci., 2015, 450, 374-380.

7 D. Nayak, S. Pradhan, S. Ashe, P. R. Rauta and B. Nayak, J. Colloid Interface Sci., 2015, 457, 329-338.
8 K. Rajaram, D. C. Aiswarya and P. Sureshkumar, Mater. Lett., 2015, 138, 251-254.

9 R. P. Raman, S. Parthiban, B. Srinithya, V. Vinod Kumar, S. P. Anthony, A. Sivasubramanian and M. S. Muthuraman, Mater. Lett., 2015, 400-403.

10 M. Ramar, B. Manikandan, P. N. Marimuthu and T. Raman, Spectrochim. Acta, Part A, 2015, 140, 223-228.

11 P. S. Ramesh, T. Kokila and D. Geetha, Spectrochim. Acta, Part A, 2015, 142, 339-343.

12 M. K. Swamy, M. S. Akhtar, S. K. Mohanty and U. R. Sinniah, Spectrochim. Acta, Part A, 2015, 151, 939-944.

13 P. Velmurugan, M. Choa, S. S. Lim and S. K. Seo, Mater. Lett., 2015, 138, 272-275.

14 O. V. Kharissova, H. V. R. Dias, B. I. Kharisov, B. O. Pérez and V. M. J. Pérez, Trends Biotechnol., 2013, 31, 240-248.

15 V. Makarov, A. Love, O. Sinitsyna, S. Makarova, I. Yaminsky, M. Taliansky and N. Kalinina, Acta Naturae, 2014, 6, 35-44. 16 A. Waheed, J. Barker, S. J. Barton, C. P. Owen, S. Ahmed and M. A. Carew, Eur. J. Pharm. Sci., 2012, 47, 464-473.

17 S. Saleem, L. Jafri, I. u. Haq, L. C. Chang, D. Calderwood, B. D. Green and B. Mirza, J. Ethnopharmacol., 2014, 156, 26-32.

18 B. K. Rasool, N. G. Shehab, S. A. Khan and F. A. Bayoumi, Pak. J. Pharm. Sci., 2014, 27, 73-81.

19 I. Bagban, S. Roy, A. Chaudhary, S. Das, K. Gohil and K. Bhandari, Asian Pac. J. Trop. Biomed., 2012, 2, S1457S1460.

20 T. Khan, B. H. Abbasi and M. A. Khan, J. Photochem. Photobiol., B, 2018, 185, 153-160.

21 T. Khan, B. H. Abbasi, M. A. Khan and Z. K. Shinwari, Appl. Biochem. Biotechnol., 2016, 179, 46-58.

22 Z.-u.-R. Mashwani, M. A. Khan, T. Khan and A. Nadhman, Adv. Colloid Interface Sci., 2016, 234, 132-141.

23 P. Das, K. Ghosal, N. K. Jana, A. Mukherjee and P. Basak, Mater. Chem. Phys., 2019, 228, 310-317.

24 K. M. Soto, C. T. Quezada-Cervantes, M. HernándezIturriaga, G. Luna-Bárcenas, R. Vazquez-Duhalt and S. Mendoza, LWT-Food Sci. Technol., 2019, 103, 293-300.

25 I. M. Chung, I. Park, K. Seung-Hyun, M. Thiruvengadam and G. Rajakumar, Nanoscale Res. Lett., 2016, 11, 40.

26 M. Ali, B. Kim, K. D. Belfield, D. Norman, M. Brennan and G. S. Ali, Mater. Sci. Eng. C, 2016, 58, 359-365.

27 I. Wiegand, K. Hilpert and R. E. W. Hancock, Nat. Protoc., 2008, 3, 163-175.

28 J. M. Pérez, I. L. Calderón, F. A. Arenas, D. E. Fuentes, G. A. Pradenas, E. L. Fuentes, J. M. Sandoval, M. E. Castro, A. O. Elías and C. C. Vásquez, PloS One, 2007, 2, e211.

29 S. Anjum and B. H. Abbasi, Int. J. Nanomed., 2016, 11, 715728.

30 S. J. Oldenburg, Silver Nanoparticles: Properties and Applications, https://www.sigmaaldrich.com/technicaldocuments/articles/materials-science/nanomaterials/silvernanoparticles.html, accessed May 30, 2020.

31 M. Wuithschick, B. Paul, R. Bienert, A. Sarfraz, U. Vainio, M. Sztucki, R. Kraehnert, P. Strasser, K. Rademann, F. Emmerling and J. Polte, Chem. Mater., 2013, 25, 46794689 . 
32 S. L. Smitha, K. M. Nissamudeen, D. Philip and K. G. Gopchandran, Spectrochim. Acta, Part A, 2008, 71, 186-190.

33 R. I. Iyer and T. Panda, J. Nanosci. Nanotechnol., 2016, 16, 7345-7357.

34 A. Nabikhan, K. Kandasamy, A. Raj and N. M. Alikunhi, Colloids Surf., B, 2010, 79, 488-493.

35 V. V. Makarov, A. J. Love, O. V. Sinitsyna, S. S. Makarova, I. V. Yaminsky, M. E. Taliansky and N. O. Kalinina, Acta Naturae, 2014, 6, 35-44.

36 M. Khan, M. Khan, S. F. Adil, M. N. Tahir, W. Tremel, H. Z. Alkhathlan, A. Al-Warthan and M. R. H. Siddiqui, Int. J. Nanomed., 2013, 8, 1507-1516.

37 S. A. Sheshadri, S. Sriram, P. Balamurugan, R. Anupriya, S. A. Princy, P. Brindha and S. Bindu, RSC Adv., 2015, 5, 47548-47554.

38 M. B. Arnao and J. Hernández-Ruiz, Agronomy, 2020, 10, 95. 39 B. Debnath, W. Islam, M. Li, Y. Sun, X. Lu, S. Mitra, M. Hussain, S. Liu and D. Qiu, Int. J. Mol. Sci., 2019, 20, 1040. 40 J. Schindelin, C. T. Rueden, M. C. Hiner and K. W. Eliceiri, Mol. Reprod. Dev., 2015, 82, 518-529.

41 T. Hartmann, Entomol. Exp. Appl., 1996, 80, 177-188.

42 M. A. Nawaz, Y. Huang, Z. Bie, W. Ahmed, R. J. Reiter, M. Niu and S. Hameed, Front. Plant Sci., 2016, 6, 1230.

43 T. Efferth, Engineering, 2019, 5, 50-59.

44 R. Farheen, B. S. Siddiqui, I. Mahmood, S. U. Simjee and S. Majeed, J. Chem. Soc. Pak., 2016, 38.

45 G. Marslin, K. Siram, Q. Maqbool, R. K. Selvakesavan, D. Kruszka, P. Kachlicki and G. Franklin, Materials, 2018, 11, 940 .
46 N. Sahu, D. Soni, B. Chandrashekhar, D. B. Satpute, S. Saravanadevi, B. K. Sarangi and R. A. Pandey, Int. Nano Lett., 2016, 6, 173-181.

47 L. Beutin, J. Vet. Med., Ser. B, 2006, 53, 299-305.

48 E. J. Bottone, Clin. Microbiol. Rev., 2010, 23, 382.

49 A. M. Brunings and D. W. Gabriel, Mol. Plant Pathol., 2003, 4, 141-157.

50 C. E. White and S. C. Winans, Philos. Trans. R. Soc., B, 2007, 362, 1135-1148.

51 R. Loria, J. Kers and M. Joshi, Annu. Rev. Phytopathol., 2006, 44, 469-487.

52 K. S. Bell, M. Sebaihia, L. Pritchard, M. T. G. Holden, L. J. Hyman, M. C. Holeva, N. R. Thomson, S. D. Bentley, L. J. C. Churcher, K. Mungall, R. Atkin, N. Bason, K. Brooks, T. Chillingworth, K. Clark, J. Doggett, A. Fraser, Z. Hance, H. Hauser, K. Jagels, S. Moule, H. Norbertczak, D. Ormond, C. Price, M. A. Quail, M. Sanders, D. Walker, S. Whitehead, G. P. C. Salmond, P. R. J. Birch, J. Parkhill and I. K. Toth, Proc. Natl. Acad. Sci. U. S. A., 2004, 101, 11105. 53 A. Basset, R. S. Khush, A. Braun, L. Gardan, F. Boccard, J. A. Hoffmann and B. Lemaitre, Proc. Natl. Acad. Sci. U. S. A., 2000, 97, 3376-3381.

54 V. R. Netala, V. S. Kotakadi, V. Nagam, P. Bobbu, S. B. Ghosh and V. Tartte, Appl. Nanosci., 2015, 5, 801-807.

55 Q. H. Xia, Y. J. Ma and J. W. Wang, Nanomaterials, 2016, 6, 160.

56 S. Dwivedi, R. Wahab, F. Khan, Y. K. Mishra, J. Musarrat and A. A. Al-Khedhairy, PloS One, 2014, 9, e111289. 